\title{
Core excitation effects in halo nuclei using a transformed oscillator basis
}

\author{
J. A. Lay*, J. M. Arias*, J. Gómez-Camacho*,† and A. M. Moro* \\ ${ }^{*}$ Departamento de FAMN, Facultad de Física, Universidad de Sevilla, Apdo. 1065, E-41080 \\ Sevilla, Spain \\ ${ }^{\dagger}$ Centro Nacional de Aceleradores, Avda. Thomas A. Edison, E-41092, Sevilla, Spain
}

\begin{abstract}
A recent generalization of the Transformed Harmonic Oscillator basis, intended to consider core excitations in the structure of one nucleon halo nuclei, is applied to the break up of ${ }^{11} \mathrm{Be}$. The reaction studied is ${ }^{11} \mathrm{Be}+{ }^{208} \mathrm{~Pb}$ at $69 \mathrm{MeV} /$ nucleon. The experimental set up is designed to ensure pure dipole Coulomb excitations. Making use of the Equivalent Photon Method and the electromagnetic transition probabilities obtained with the transformed oscillator basis, a relevant contribution of the quadrupole excitations of the core is found. The inclusion of core excitations is, therefore, necessary for the correct extraction of the dipole electromagnetic transition probability of halo nuclei.
\end{abstract}

Keywords: nuclear reactions; halo nuclei; breakup; core excitation

PACS: $24.50 .+\mathrm{g}, 25.60 . \mathrm{Gc}, 27.20 .+\mathrm{n}, 21.60 . \mathrm{Gx}$

During the last few years, reactions involving loosely bound exotic nuclei, such as halo nuclei, have been one of the most active fields in Nuclear Physics. This kind of reactions is known to be strongly influenced by the coupling to the continuum (i.e., unbound) states. For two-body projectiles, a successful technique to describe these processes is the Continuum-Discretized Coupled-Channels (CDCC) method [1]. Here the continuum can be described in terms of energy or momentum "bins" (the average method) or, alternatively, by the eigenstates of the two-body Hamiltonian in a basis of square-integrable functions, or pseudo-states (PS).

In this contribution a PS basis obtained performing a simple analytic local scale transformation (LST) to the harmonic oscillator basis (THO) is used. This THO basis is easy to calculate and reproduces a wide variety of observables, requiring a small number of functions compared to other bases [2]. Moreover, narrow resonances are well characterized by one or two PS.

Recently, we have extended this basis to describe systems with a valence + core structure (such as the halo nuclei), taking into account the possible excitations of the core [3]. In this new framework, the PS of the projectile is a combination of core states and valence configurations coupled to the corresponding total angular momentum. The spectroscopic factors for each contribution will be determined by the Hamiltonian proposed to describe the interaction between core and valence. Most of the cores in the ${ }^{11} \mathrm{Be}$ and odd A carbon isotopes region are deformed. Therefore, the interaction between core and valence can be modeled with a particle-rotor Hamiltonian [3].

We have applied this model to the ${ }^{11} \mathrm{Be}+{ }^{208} \mathrm{~Pb}$ reaction at $69 \mathrm{MeV} /$ nucleon comparing with the available data from [4]. At this high energy and within the angular range studied, the break up is expected to be due to pure Coulomb excitations. Therefore, it is

La Rabida 2012 International Scientific Meeting on Nuclear Physics AIP Conf. Proc. 1541, 152-153 (2013); doi: 10.1063/1.4810823 (C) 2013 AIP Publishing LLC 978-0-7354-1165-4/\$30.00 


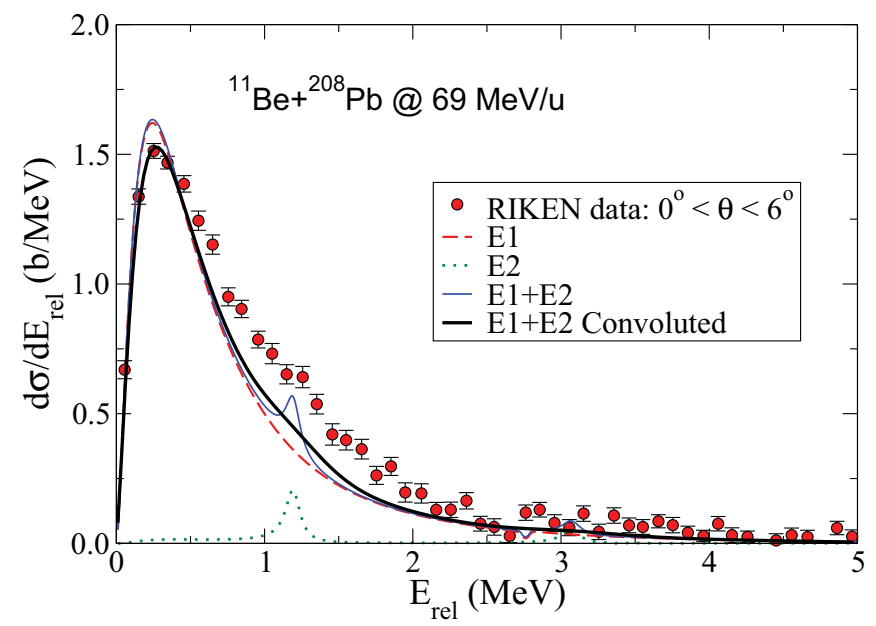

FIGURE 1. (Colour online) Energy distribution of the exclusive breakup for the reaction ${ }^{11} \mathrm{Be}+{ }^{208} \mathrm{~Pb}$ at $69 \mathrm{MeV} /$ nucleon. The circles are the experimental data from Ref. [4]. The dashed and dotted lines correspond to the $E 1$ and $E 2$ contributions, calculated within the EPM method. The thin solid line is the sum of both contributions and the thick solid line is this sum convoluted with the experimental resolution from [4].

possible to apply the semiclassical Equivalent Photon Method [5] where the cross section is directly connected to the corresponding electromagnetic transition probabilities $\mathscr{B}(E \lambda)$. In a single-particle model based on an inert core, quadrupole, $E 2$, transitions are negligible so that the break up is given by the dipole, $E 1$, transition. However, the excitations of the core give rise to an important quadrupole contribution that improves the agreement with the data as seen in Fig. 1. These experiments are used to extract $\mathscr{B}(E 1)$ energy distribution of halo nuclei. Therefore, an appropriate treatment of core excitations is necessary for a correct analysis of these reactions and the extraction of reliable electromagnetic transition probabilities.

\section{ACKNOWLEDGMENTS}

This work has been partially supported by the Spanish Ministerio de Ciencia e Innovación and FEDER funds under Projects No. FIS2011-28738-c02-01, No. FPA200907653, and No. FPA2009-08848, by the Spanish Consolider-Ingenio 2010 Programme CPAN (CSD2007-00042), and by Junta de Andalucía (FQM160, P07-FQM-02894). J.A.L. acknowledges a research grant by the Ministerio de Ciencia e Innovación.

\section{REFERENCES}

1. N. Austern, et al., Phys. Rep. 154, 125 (1987).

2. J. A. Lay, A. M. Moro, J. M. Arias, and J. Gómez-Camacho, Phys. Rev. C 82, 024605 (2010).

3. J. A. Lay, A. M. Moro, J. M. Arias, and J. Gómez-Camacho, Phys. Rev. C 85, 054618 (2012).

4. N. Fukuda, et al., Phys. Rev. C 70, 054606 (2004).

5. C. A. Bertulani, and G. Baur, Phys. Rep. 163, 299 (1988). 